\title{
El Mapa Integrado Andino del Norte
}

\author{
Ángel Martín, Juan José Contreras, Edson Salinas y Percy Valverde* \\ Rafael Balbi y Vinicius E. Medeiros** \\ Alesandra Vianey y Amadeo Fajardo*** \\ Paulina Guerrón y Eliana Tene \\ Elizabeth Sámuels y Ariel Agrazal ${ }^{\star}$ \\ Reynaldo Flores y Wilman Avilés $\bullet$ \\ Jean Parcher y Roberto Lugo ${ }^{+}$ \\ Luis Miguel Blanco y Antonio F. Rodríguez ${ }^{+}$
}

Recibido el 28 de noviembre de 2016; aceptado el 30 de enero de 2017

\begin{abstract} ama, Colombia, Ecuador, Peru and Bolivia.

* Instituto Geográfico Militar (IGM Bolivia).

** Instituto Brasileño de Geografía y Estadística (IBGE).

*** Instituto Geográfico Agustín Codazzi de Colombia (IGAC).

- Instituto Geográfico Militar (IGM Ecuador).

- Instituto Geográfico Nacional Tommy Guardia de Panamá (IGNTG).

- Instituto Geográfico Nacional (IGN Perú).

- Servicio Geológico de Estados Unidos (USGS).

+ Centro Nacional de Información Geográfica (CNIG, España).
\end{abstract}

The Integrated North Andean Map, is an initiative led by the Pan American Institute for Geography and History (PAIGH) and supported by the CAF/Latin American Development Bank, in the context of the GeoSUR Program. The aim of this initiative is to develop the first Integrated Map of the countries of the North Andean area at 1:250,000 scale through the coordinated work of the Geographic Institutes of Pan-

This initiative also receives the specialized guidance and technical coordination provided by the National Centre for Geographic Information (CNIG) of the National Geographic Institute of Spain. In addition, the National Section of the PAIGH in the United States of America has made special contributions through the International Technical Assistance Program of the Department of the Interior (DOI-ITAP) and the United States Agency for International Development (USAID); there has also 
been the specialized advice of the Geological Service of the United States (USGS).

The map is intended to benefit all of the geographic information users in the countries of the Community of Andean Nations by means of making available and providing access to quality regional cartographic information that is oficial, integrated and harmonized at supranational level.

This project integrates the main feature types of Hydrography, Administrative Boundaries, Settlements and urban areas, Transport networks, Land Morphology and some elements considered as indispensable to understanding the geography of each country, grouped under the title Miscellaneous.

A participative methodology makes it possible not only to integrate technical criteria for the geospatial information of five countries, but also to ensure continuity for this Project by means of the commitment and integration between the official Geographic Institutes of each country, at a scale that is useful for regional planning and territorial organization.

Among the potential uses of this information, one can mention planning for development projects, environmental studies, infrastructure, environmental impact, management of natural disasters, climate change studies and projects for crossfrontier integration, among others.

The expectation for the future of this map is to integrate it with the Integrated Map of Centroameric and then, with the Integrated Map of the rest of South America.

Key words: Central America Integrated, Andean, PAIGH, CAF, GeoSUR, Panama, Colombia, Ecuador, Peru and Bolivia.

\section{Resumo}

O Mapa Integrado Andino do Norte, é uma iniciativa liderada pelo Instituto Panamericano de Geografia e História (IPGH) e apoiada pelo Banco de Desenvolvimento da América Latina (CAF), no contexto do Programa GeoSUR, que tem por objeto desenvolver o Primero Mapa Integrado dos países Andinos do Norte na escala 1:250,000 através do trabalho coordenado dos Institutos Geográficos do Panamá, Colômbia, Equador, Peru e Bolívia.

Esta iniciativa contou com a assessoria especializada e coordenação técnica do Centro Nacional de Informação Geográfica (CNIG) do Instituto Geográfico Nacional da Espanha e com a contribuição especial da Seção Nacional do IPGH nos Estados Unidos da América, através do Programa de Assistência Técnica Internacional do Departamento do Interior (DOI-ITAP), da Agência dos Estados Unidos para o Desenvolvimento Internacional (USAID) e, com a assessoria do Serviço Geológico dos Estados Unidos (USGS). 
O mapa tem por finalidade beneficiar o conjunto de usuários de informação geográfica dos países da Comunidade Andina das Nações através da disponibilização e acesso a informação cartográfica regional oficial, de qualidade, integrada e harmonizada a nível supranacional.

Este projeto integra os principais elementos da Hidrografia, Limites Administrativos, Povoados, Vias, Morfologia do Terreno e alguns elementos considerados como indispensáveis para entender a geografia de cada país, agrupados sob o título de Miscelâneas.

Uma metodologia participativa permite integrar não somente critérios técnicos da informação geoespacial de cinco países, também permite assegurar a continuidade deste projeto através do compromisso e integração dos Institutos Geográficos oficiais de cada país, a uma escala útil para o planejamento regional e o ordenamento do território.

Dentre os potenciais usos desta informação se pode mencionar o planejamento de projetos de desenvolvimento, estudos ambientais, infraestrutura, impacto ambiental, prevenção de desastres naturais, estudos de mudança climática e projetos de integração fronteiriça, entre outros.

A perspectiva deste mapa no futuro, é integrá-lo ao mapa da América Central e posteriormente ao resto dos países da América do Sul.

Palavras chave: Integrado, Andino, IPGH, CAF, GeoSUR, Panamá, Colômbia, Equador, Peru e Bolívia.

\section{Resumen}

El Mapa Integrado Andino del Norte, es una iniciativa liderada por el Instituto Panamericano de Geografía e Historia (IPGH) y apoyada por CAF-Banco de Desarrollo de América Latina, en el contexto del Programa GeoSUR, que tiene por objeto desarrollar el Primer Mapa Integrado de los países Andinos del Norte a escala 1:250.000 a través del trabajo coordinado de los Institutos Geográficos de Panamá, Colombia, Ecuador, Perú y Bolivia.

Esta iniciativa contó con la asesoría especializada y coordinación técnica del Centro Nacional de Información Geográfica (CNIG) del Instituto Geográfico Nacional de España y con la contribución especial de la Sección Nacional del IPGH en Estados Unidos de América, a través del Programa de Asistencia Técnica Internacional del Departamento del Interior (DOI-ITAP), de la Agencia de los Estados Unidos para el Desarrollo Internacional (USAID) y con la asesoría del Servicio Geológico de Estados Unidos (USGS).

El mapa tiene por finalidad beneficiar al conjunto de usuarios de información geográfica de los países de la Comunidad Andina de Naciones a través de la disponibilidad y acceso a información cartográfica regional oficial, de calidad, integrada $\mathrm{y}$ armonizada a nivel supranacional. 
Este proyecto integra los principales elementos de la Hidrografía, Límites Administrativos, Poblados, Vías, Morfología del Terreno y algunos elementos considerados como indispensables para entender la geografía de cada país, agrupados bajo el titulo Miscelánea.

Una metodología participativa permite integrar no solo criterios técnicos de la información geoespacial de cinco países, también permite asegurar la continuidad de este proyecto a través del compromiso e integración de los Institutos Geográficos oficiales de cada país, a una escala útil para la planificación regional y el ordenamiento del territorio.

Entre los potenciales usos de esta información se puede mencionar la planificación de proyectos de desarrollo, estudios ambientales, infraestructura, impacto ambiental, prevención de desastres naturales, estudios de cambio climático y proyectos de integración fronteriza, entre otros.

La proyección futura de este mapa es unirlo con el Mapa Integrado de Centroamérica y en el futuro con el Mapa Integrado de todo Sudamérica.

Palabras clave: Integrado, Andino, IPGH, CAF, GeoSUR, Panamá, Colombia, Ecuador, Perú y Bolivia.

\section{Antecedentes}

Los innumerables eventos y desastres naturales que han venido aconteciéndose en las diferentes partes del mundo han tenido grandes repercusiones desde el punto de vista económico, ambiental y social.

En ese contexto, disponer de información confiable y oportuna es la base fundamental para una adecuada planificación preventiva y una gestión eficaz de la situación posterior, es aquí en donde la geoinformación se convierte en un instrumento básico y fundamental para que los países puedan realizar una adecuada toma de decisiones.

Un desastre natural no tiene límites políticos ni administrativos, su afectación traspasa fronteras y los fenómenos que lo provocan son trasnacionales; por tal motivo, la información requerida para su gestión es de escala global y se necesita una amplia participación de todos quienes habitamos este planeta.

Esa información requerida es de diferente índole y temática, pero en cualquier caso se construye sobre la cartografía básica, la cual es generada de manera oficial por los institutos cartográficos o geográficos de cada país. Lograr un mapa base unificado a nivel mundial era un objetivo que perseguía la iniciativa internacional del Mapa Global y nuestra región contribuyó a través del Grupo de Trabajo del Mapa Global de las Américas (MGA) en el 2005, a la escala de trabajo 1:1.000.000. Este objetivo fue alcanzado parcialmente a través del proyecto "Integración de Datos Mapa Global de Sudamérica" que fue concretado a fines del año 2012 (Hunt, 2012). 
Una mayor escala con un mayor grado de detalle fue el siguiente reto, esta necesidad fue presentada inicialmente a través de una solicitud al Instituto Panamericano de Geografía e Historia (IPGH) en el 2009 y se plasmó en la iniciativa del "Mapa Integrado de Centroamérica" a escala 1:250,000 (Norori y otros, 2013) (véase la Figura 1).

En la ejecución de este proyecto se desarrollaron cuatro talleres técnicos llevados a cabo desde el 2011 hasta el 2014, contando con la participación de los institutos geográficos, cartográficos, centros y direcciones nacionales responsables de la cartografía oficial de los países de Centroamérica y de México, se obtuvo como resultado un mapa digital integrado con la cartografía base de toda esa región: sur de México, Belice, Guatemala, Honduras, El Salvador, Nicaragua, Costa Rica y Panamá, con la cooperación del Servicio Geológico de los Estados Unidos (USGS) y el apoyo de la CAF-Banco de Desarrollo de América Latina.

La información cartográfica básica obtenida de ese proyecto fue: poblados, mancha urbana, límites administrativos, carreteras, ríos y cuerpos de agua.

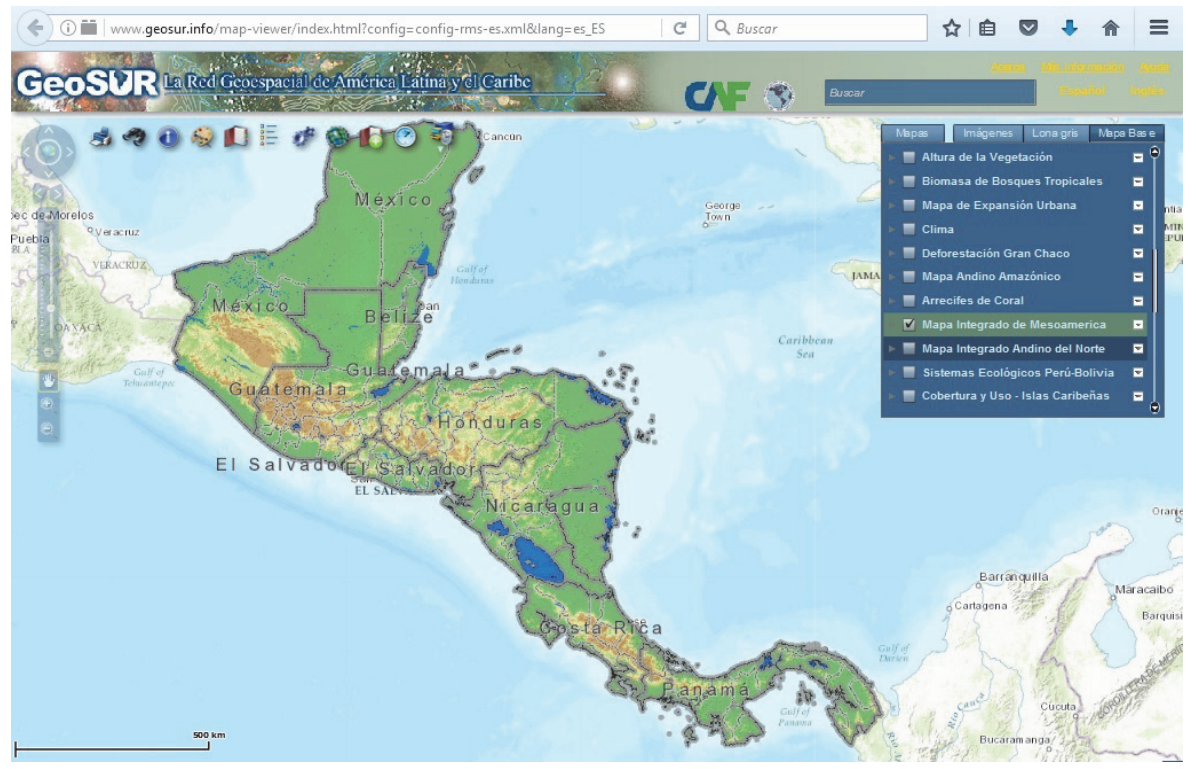

Figura 1. Mapa Integrado de Centroamérica.

Siguiendo con esa iniciativa en el 2014 nace el proyecto "Mapa Integrado Andino del Norte (MIAN)", con la participación de los Institutos Geográficos de la región norte de Suramérica: Panamá como enlace con el proyecto "Mapa Integrado de Centroamérica", Colombia, Ecuador, Perú y Bolivia. Para este proyecto se contó con la asesoría y asistencia técnica del Centro Nacional de Información Geográfica 
de España (CNIG), la cooperación del USGS y el auspicio de la CAF-Banco de Desarrollo de América Latina.

Para la ejecución del MIAN se desarrollaron tres talleres técnicos en el 2015, y un taller intermedio, con la participación del Instituto Geográfico Nacional Tommy Guardia de Panamá, el Instituto Geográfico Agustín Codazzi de Colombia, el Instituto Geográfico Militar de Ecuador, el Instituto Geográfico Nacional de Perú y el Instituto Geográfico Militar de Bolivia. En el tercer taller se obtuvo como resultado una primera versión del Mapa Integrado de estos países a escala 1:250,000, con la información de poblados, mancha urbana, límites administrativos, carreteras, ríos, cuerpos de agua, formas hidrográficas, línea de ferrocarril, islas.

En mayo de 2016, se desarrolló el cuarto taller del MIAN, en el cual se incorporó el Instituto Brasileño de Geografía y Estadística (IBGE), como una iniciativa para el enlace con el nuevo proyecto de "Mapa Integrado de América del Sur" (MIAS), el cual tuvo también la colaboración del CNIG y en el tema del grafo de hidrografía la asistencia técnica del USGS.

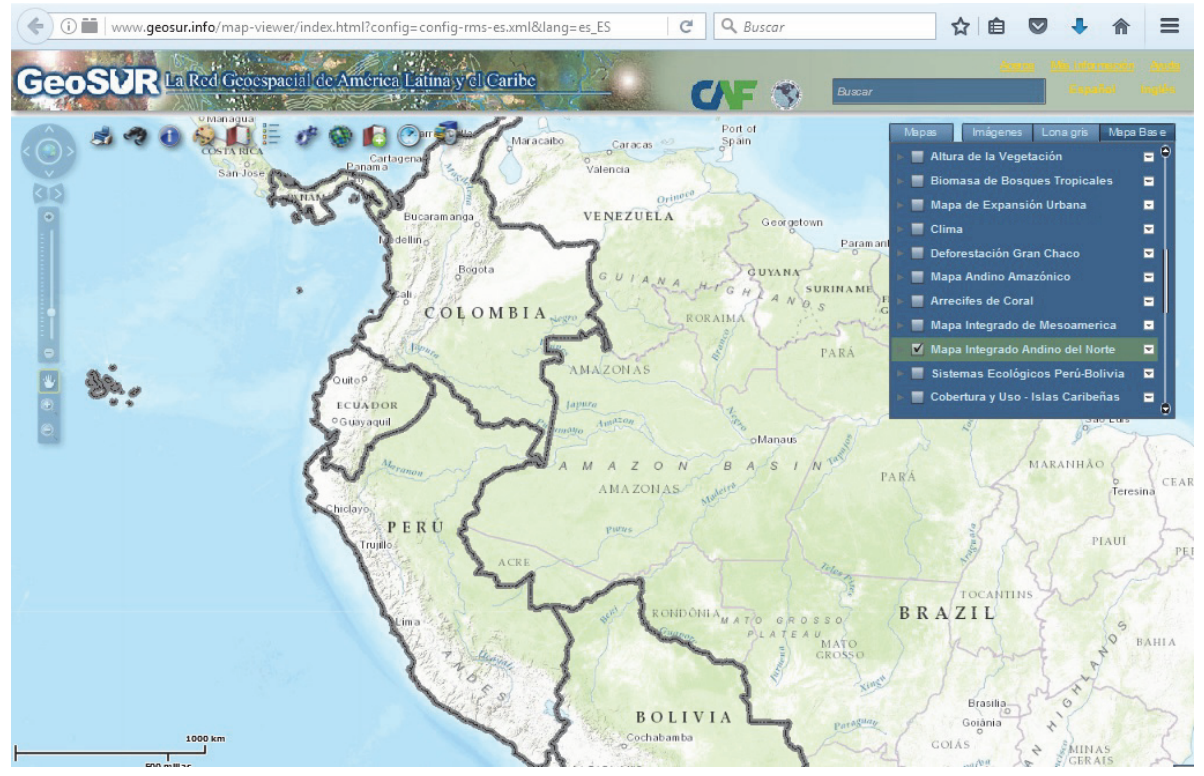

Figura 2. El WMS MIAN en el Visualizador regional del Programa GeoSUR.

Con los resultados obtenidos del "Mapa Integrado de Centroamérica" y la primera versión obtenida del "Mapa Integrado Andino del Norte", ya se tiene disponible una base cartográfica consensuada, continua, armonizada y oficial de 12 países 
de Centro y Suramérica, generada por los organismos rectores de la cartografía en sus respectivos países.

El servicio WMS de la base cartográfica del MIAN (Rodríguez, 2016) está disponible en el Visualizador regional del Programa GeoSUR (véase la Figura 2) y tiene una licencia consensuada por los países miembros del tipo CC BY 4.0, la cual permite un libre uso y requiere la mención de la fuente de la siguiente forma: $\mathrm{CC}$ BY 4.0 CAF, IPGH, Programa GeoSUR, IGM Bolivia, IGAC Colombia, IGM Ecuador, IGTG Panamá, IGN Perú.

Todas las iniciativas plasmadas y ejecutadas a través de los proyectos MI (Mapas Integrados) de Centroamérica, MIAN y MIAS, conllevan al cumplimiento de un objetivo macro, la conformación y generación del Mapa de las Américas.

\section{Objetivos}

\section{Objetivo general}

El objetivo general y esencial del proyecto MIAN es producir de manera colaborativa el primer mapa digital integrado de datos geoespaciales a escala 1:250.000 basado en datos oficiales de los países Andinos del Norte, Bolivia, Colombia, Ecuador y Perú, y como enlace con Centroamérica, Panamá.

El concepto de integrado quiere decir que se trata de una mapa digital con continuidad geométrica y semántica, con un modelo único y sin costuras (seamless), es decir que no presenta diferencias técnicas al pasar de un país a otro más allá de las que se derivan de diferencias en el mundo real.

\section{Objetivos específicos}

- Desarrollar talleres técnicos de integración y empalme de los datos geoespaciales oficiales a escala 1:250.000, proporcionados por los Institutos Geográficos de cada país participante.

- Consensuar acuerdos referentes a: Especificaciones Técnicas, Catálogo de Objetos, Modelo UML, Política de Datos, Metadatos, Licencia de uso, entre otros.

- Producir documentación técnica complementaria y normalizada: especificaciones de producto, catálogo de objetos, catálogos de representación (Proyecto MIAN, 2016) y documentación (artículos, presentaciones, etc.).

- Llevar a cabo una campaña de difusión del proyecto MIAN en blogs, redes sociales y listas de correo, además de eventos presenciales. 
- Publicar las bases cartográficas oficiales generadas y consensuadas en el Proyecto MIAN, unificadas en un único conjunto de datos a través de un servicio WMS visible en el Visualizador Regional del Programa GeoSUR.

\section{Desarrollo de los talleres participativos}

En los talleres del proyecto MIAN han participado regularmente el doctor Rodrigo Barriga (IPGH), el doctor Santiago Borrero (CAF y GeoSUR), el ingeniero Roberto Lugo (USGS), los ingenieros Antonio F. Rodríguez y Luis Miguel Blanco (CNIG) y técnicos especialistas de los Geoinstitutos implicados (véase la Figura 3):

- Instituto Geográfico Militar (Bolivia)

- Instituto Geográfico Agustín Codazzi (Colombia)

- Instituto Geográfico Militar (Ecuador)

- Instituto Geográfico Nacional Tommy Guardia (Panamá)

- Instituto Geográfico Nacional (Perú)

Además, han colaborado los directores de los Institutos implicados en el Primer Taller, el Director del Instituto anfitrión en cada caso, designó personal de apoyo, al comité organizador local y expertos adicionales, y por último, en el Cuarto Taller se tuvo una nutrida representación del IBGE de Brasil.
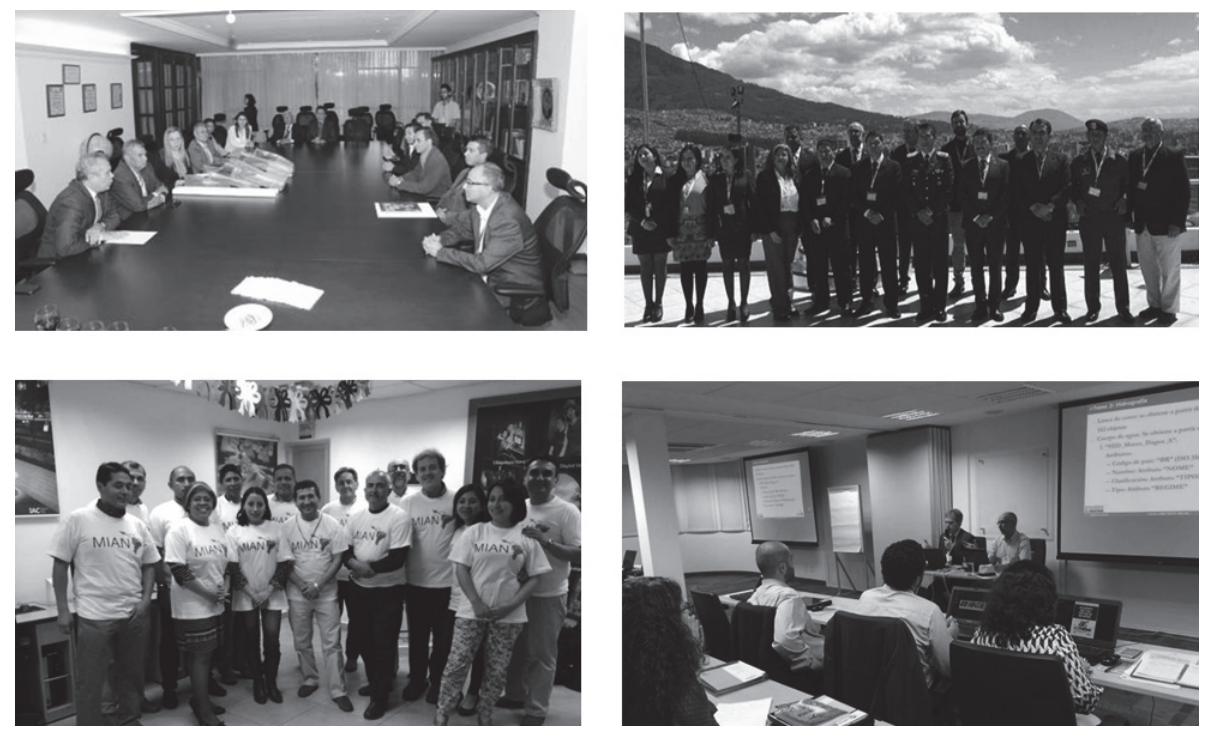

Figura 3 De izquierda a derecha y de arriba abajo, $1^{\circ}, 2^{\circ}, 3^{\circ}$ y $4^{\circ}$ Taller MIAN. 


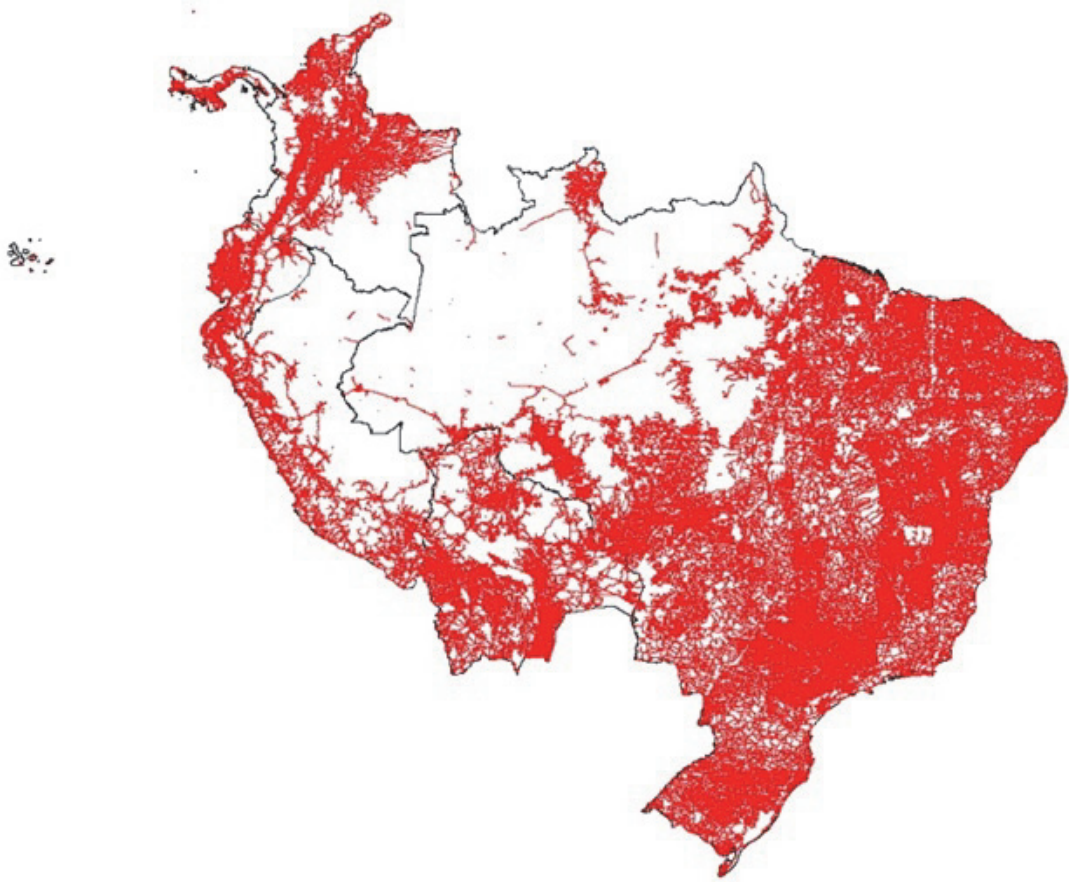

Figura 4. Carreteras de los países MIAN y Brasil.

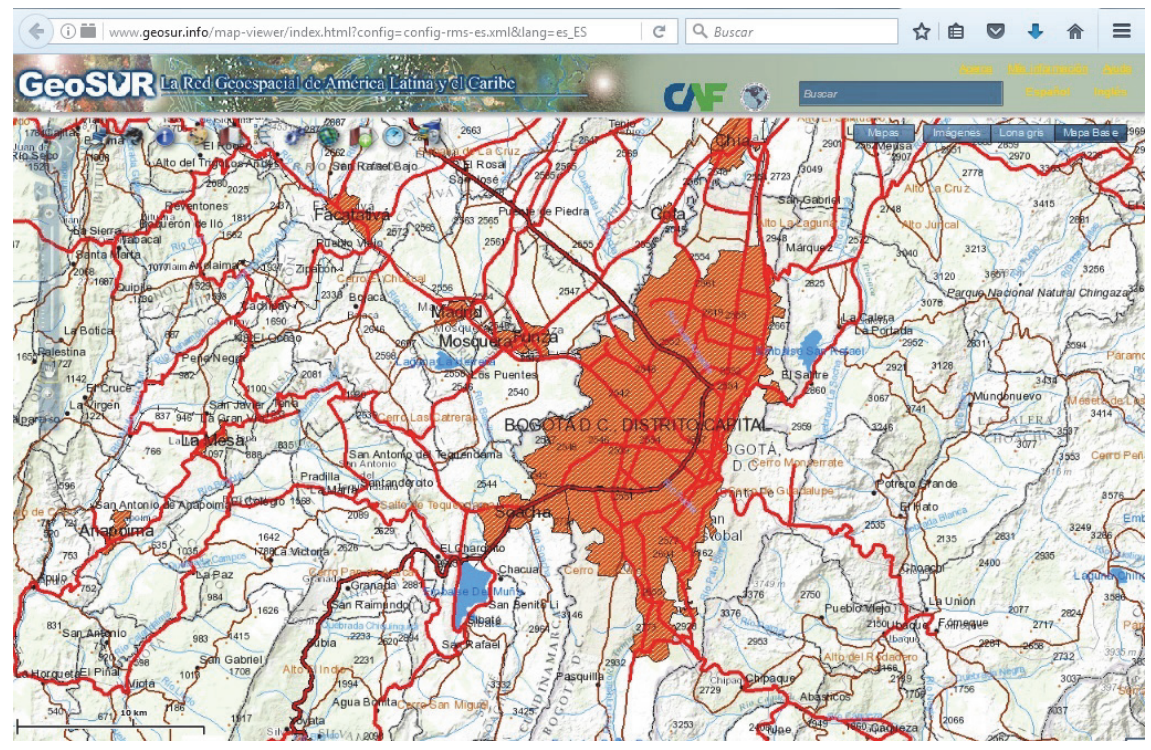

Figura 5. Web Map Service del Mapa Integrado Andino del Norte. 


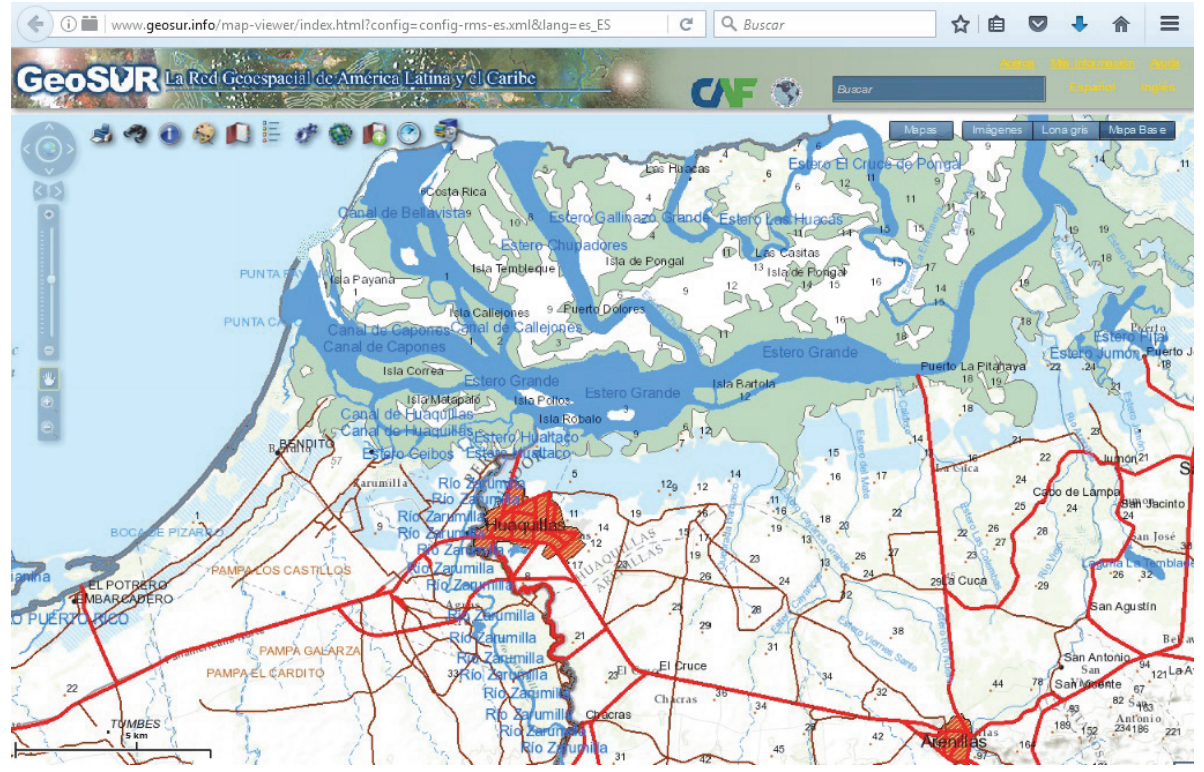

Figura 6. Visualización del Mapa integrado Andino del Norte.

Se han llevado a cabo cuatro talleres de trabajo práctico conjunto de una semana de duración, de lunes a viernes, entre seis y siete horas de trabajo al día, con una reunión previa de coordinación el domingo anterior, excepto en el Cuarto Taller que se celebró el mismo lunes por la mañana, al que asistieron los participantes del IPGH, GeoSUR, USGS, CNIG y el comité organizador local.

Adicionalmente se ha celebrado un taller intermedio de dos días de duración.

\section{Primer Taller}

Se celebró del 9 al 13 de febrero de 2015 en la sede del IGAC en Bogotá, Colombia.

Como preparación previa al taller, el CNIG había distribuido una encuesta para conocer en detalle las especificaciones de los datos que cada país aportaría al proyecto. En este Taller de arranque se contó con la presencia y el compromiso directo de los directores de los Geoinstitutos participantes. Se cohesionó un equipo de trabajo multinacional y cada país expuso las características principales de su cartografía.

Como resultados, se definió el marco general de trabajo, se consensuaron las decisiones más importantes: una primera versión de especificaciones y de Catálogo de objetos. Se comenzó el trabajo de case de contornos de país y de Hidrografía. Se consensuó un programa de trabajo para seguir avanzando. 


\section{Taller Intermedio}

Tuvo lugar el 7 y 8 de abril de 2015 en las oficinas del Instituto Geográfico Nacional (IGN) de Perú, Lima. Los objetivos de este taller eran dos, por un lado incorporar al Instituto Geográfico Militar de Bolivia, que no asistió al Primer Taller por causas de fuerza mayor, consensuando los objetivos, las especificaciones generales, el plan de trabajo y la filosofía general, y empezar a trabajar en los cases entre Bolivia y Perú.

Se incorporó al Instituto Geográfico Militar de Bolivia a los consensos generados en el Primer taller, se elaboró un calendario de entregas de datos para esta institución y se iniciaron los trabajos de case.

\section{Segundo Taller}

Celebrado del 13 al 17 de julio de 2015 en la sede del Instituto Geográfico Militar (IGM) de Ecuador. Se perfiló un Catálogo prácticamente definitivo de objetos geográficos, estructurado en temas, objetos y atributos, un modelo conceptual en UML y se progresó notablemente en la finalización de los contornos de país y los cases de información, con lo que quedaron una serie de problemas puntuales cuya resolución quedó planificada antes del siguiente taller.

Se resolvieron las incidencias encontradas en los procesos de chequeo ejecutado por el CNIG, se planteó e inició la generación de los ejes de la Hidrografía y hubo una primera sesión de exposición de la metodología de generación del grafo de Hidrografía a cargo del ingeniero Roberto Lugo (USGS).

\section{Tercer Taller}

Tuvo lugar del 16 al 20 de noviembre de 2015, en oficinas coordinadas por el Instituto Geográfico Nacional Tommy Guardia (IGNTG) en la Ciudad de Panamá, Panamá.

Se corrigieron los problemas de detalle pendientes en cuanto a case de la información, y se trabajó intensamente en el refinamiento de los datos, corrigiendo las incidencias detectadas por el CNIG en sus rutinas de chequeo.

Se aprobaron una serie de decisiones y recursos: llegando a un consenso definitivo del Catálogo de Objetos Geográficos, el Modelo UML y las especificaciones de producto; se hizo una práctica de generación de metadatos conforme a LAMP v1.0 (versión 1 del Perfil Latinoamericano de Metadatos); se acordó adoptar como simbología de representación la adoptada como norma por el IPGH plasmada en dos documentos aportados por el IGM de Ecuador; se determinó no considerar las curvas de nivel, toda vez que completar el case para ese objeto constituía en varios casos un trabajo excesivo, se decidió utilizar el SRTM, gratuito y homogéneo. El tema de Morfología del Terreno queda reducido a Puntos acotados. 
Se consensuó como periodo de actualización cinco años y adoptar una licencia CC BY (reconocimiento) 4.0 para el servicio WMS. Por último se eligió un editor para la redacción de un artículo para la Revista del IPGH y se repartió el trabajo.

\section{Cuarto Taller}

El cuarto y último taller del proyecto MIAN se celebró del 16 al 20 de mayo de 2016 en la sede del Instituto Brasileño de Geografía y Estadística (IBGE) en Río de Janeiro, Brasil. En él se abordaron tres líneas de trabajo: finalizar todos los detalles pendientes para publicar el WMS MIAN; avanzar en el grafo de Hidrografía, hasta el punto de que varios países obtuvieron un primer borrador, e incorporar a Brasil al proyecto, casando los contornos de país y progresando en el resto de temas, con la complejidad que supone al suponer un país de más de 8.5 millones de $\mathrm{km}^{2}$ de superficie, una red hidrográfica muy densa y unos $14,700 \mathrm{~km}$ de frontera.

Los cinco talleres fueron un éxito y en todos ellos se alcanzaron los objetivos previstos, contando con la compleción de algunas tareas en los períodos entre talleres y, lo que es más importante, se ha formado un equipo de trabajo en el que los técnicos de los países implicados han trabajado juntos en una atmósfera de sana colaboración y cooperación.

\section{Metodología}

\section{Datos de partida}

La mayoría de los cinco institutos geográficos participantes en el proyecto aportaron datos geográficos digitales a escala 1:250.000, excepto Perú y Colombia que disponían de datos a escala 1:100.000 que tuvieron que generalizar y simplificar en mayor o menor medida (véase la Tabla 1).

\section{Política de datos}

La dirección de los cinco institutos participantes acordó adoptar una política de datos para el Servicio Web de Mapas (WMS) basado en una licencia Creative Commons 4.0 Reconocimiento (CC BY 4.0), ${ }^{1}$ que permite todo tipo de usos con la condición de reconocer la autoría propiedad de los datos con la siguiente expresión: CC BY 4.0 CAF, IPGH, Programa GeoSUR, IGM Bolivia, IGAC Colombia, IGM Ecuador, IGNTG Panamá, IGN Perú. 
Tabla 1

\begin{tabular}{|c|c|c|c|c|}
\hline Organización & Resolución & $\begin{array}{l}\text { Proceso de } \\
\text { producción }\end{array}$ & $\begin{array}{c}\text { Fecha } \\
\text { de los datos }\end{array}$ & $\begin{array}{l}\text { Exactitud } \\
\text { estimada }\end{array}$ \\
\hline IGN Bolivia & $1: 250.000$ & $\begin{array}{l}\text { Digitalización } \\
\text { sobre Landsat } 7 \text { y } \\
\text { adaptación a } \\
\text { datos existentes }\end{array}$ & 2013 & $+50 \mathrm{~m}$ \\
\hline IGAC Colombia & $1: 250.000$ & $\begin{array}{l}\text { Generalización } \\
\text { de datos } \\
1: 100.000\end{array}$ & 2013 & $+75 \mathrm{~m}$ \\
\hline IGM Ecuador & $1: 250.000$ & $\begin{array}{l}\text { Generalización } \\
\text { 1:50.000 y carto- } \\
\text { grafía binacional } \\
\text { en fronteras }\end{array}$ & 2014 & $+50 \mathrm{~m}$ \\
\hline IGNTG Panamá & $1: 250.000$ & $\begin{array}{l}\text { Generalización } \\
1: 50.000 \mathrm{y} \\
\text { actualización }\end{array}$ & 2014 & $+30 \mathrm{~m}$ \\
\hline IGN Perú & $1: 100.000$ & $\begin{array}{l}\text { Restitución } \\
\text { fotogramétrica }\end{array}$ & 2014 & $+50 \mathrm{~m}$ \\
\hline
\end{tabular}

Fuente: Datos de cada Instituto geográfico.

Con la finalidad de maximizar su uso y publicar un servicio abierto que pueda ser explotado por cualquier usuario, con cualquier finalidad y en todo tipo de actividades y proyectos.

Por otro lado también acordaron que los contornos del objeto geográfico "País" son referenciales, orientativos, aproximados y no tienen ninguna validez oficial, ni probatoria, en consonancia con el Capítulo I, artículo 1 del Estatuto Orgánico del IPGH (IPGH, 2014).

\section{Metodología de trabajo}

Durante los talleres se han debatido todos los aspectos relevantes del proyecto hasta llegar a un consenso por unanimidad. También se han distribuido los datos en equipos multinacionales para abordar la edición de la información para su case y armonización. En todo momento, cada instituto participante ha conservado la soberanía y control sobre sus datos, de manera que todas las modificaciones realizadas han contado con su aprobación.

En los intervalos entre talleres se ha trabajado mediante teleconferencias, correo electrónico e intercambio de ficheros de datos y metadatos. El CNIG se ha encargado 
de verificar la geometría, su consistencia lógica e identificar incidencias residuales a corregir.

La metodología de trabajo se ha basado en una serie de principios generales aceptados por unanimidad:

- Debate y discusión de los aspectos clave

- Decisiones tomadas por consenso

- Conformidad con normas ISO 19100 (ISO, 2016), estándares OGC (OGC, 2016), recomendaciones del IPGH y estándares más extendidos

- Utilización de las imágenes Landsat 8 como imágenes de referencia

- Aprovechamiento de la experiencia de los facilitadores del CNIG en proyectos similares en Europa

- Aprovechamiento de la experiencia del USGS en la generación de grafos de Hidrología

- Envío de los datos al CNIG para su chequeo y verificación entre talleres

- Trabajo por aproximaciones sucesivas de calidad creciente

Los trabajos se han estructurado según una serie de tareas necesariamente secuenciales que mantienen la lógica del proceso:

1) Encuesta sobre los datos disponibles, para conocer los conjuntos de datos a integrar e identificar con la máxima antelación posibles problemas potenciales, como diferencias de resolución, divergencias de modelos, calidad diferente, actualización dispar, etc.

2) Acuerdo de unas especificaciones de producto de datos conformes a la norma ISO 19131, consensuando un primer borrador que se irá refinando según avance el proyecto. Incluye un nombre de producto, un acrónimo, una resolución de referencia, un Sistema de Referencia de Coordenadas, un Modelo UML, un Catálogo de objetos geográficos estructurado en temas, objetos y atributos, un periodo de actualización, unos metadatos, etc.

3) Selección de los datos para cada país, en un proceso que puede ser delicado cuando hay varios conjuntos disponibles en un Geoinstituto a una escala cercana a la de referencia y con distintas propiedades (unos datos más actualizados, otros con mejor geometría, etcétera) y en el que el facilitador solo debe acompañar a los técnicos en su toma de decisiones.

4) Elaboración de contornos de país únicos y comunes, teniendo en cuenta que en el MIAN se trabaja con una línea referencial y aproximada, cartográfica y orientativa, solo para dibujar el contorno del país, pero sin validez jurídica, legal, administrativa ni de ningún tipo. No se trabaja con la frontera oficial porque ese no es el objeto de este proyecto. 
5) Case de la geometría de Hidrografía, Carreteras y Poblaciones, en ese orden. En general, es la fase más trabajosa y que consume más horas-persona por la gran cantidad de situaciones que es necesario resolver de manera interactiva (véase la Figura 4).

6) Verificación de errores y chequeo. En el ínterin entre talleres, el CNIG ha procedido a ejecutar rutinas de chequeo de la geometría y topología, reportar errores y plantear a cada organización la resolución de las incidencias detectadas.

7) Corrección de errores. Se solicita a cada Geoinstituto que resuelva, en el tiempo entre talleres, los problemas encontrados para ir depurando y afinando la geometría, que habitualmente contiene errores residuales.

8) Elaboración de temas complementarios (Puntos acotados, Miscelánea...). El tema Miscelánea incluye todos los Puntos de Interés (PoI) de todo tipo que resulta inexcusable que no aparezcan en un mapa a la escala que estamos trabajando.

9) Generación de un grafo de Hidrografía con la metodología del USGS, que tiene gran experiencia en ese campo. Una vez casada y corregida de errores la geometría esta fase puede avanzar en paralelo a las demás.

10) Elaboración de metadatos, que describan conforme al Perfil Latinoamericano de Metadatos (LAMP v1.) los datos con un registro de metadatos por país, más un registro de metadatos adicional para el conjunto del MIAN; que se concibe así como una serie en la que las unidades (u hojas) son los datos de cada país que lo integran. También se catalogará el servicio WMS MIAN en cuanto esté disponible.

11) Publicación del WMS MIAN (véanse las Figuras 5 y 6), acompañado de una versión WMTS, con la simbología acordada, tarea que corre a cargo del USGS en el marco de su colaboración con el Programa GeoSUR.

\section{Conclusiones}

Puede considerarse un éxito y un activo de la máxima importancia el disponer de un mapa integrado, es decir continuo, multinacional, sin costuras y normalizado, de la zona andina del norte, disponible en forma de Servicio Web de Mapas, ${ }^{2}$ disponible en el visualizador regional ${ }^{3}$ del Programa GeoSUR, y acompañado de los recursos que completan su definición y lo hacen realmente útil, como son especificaciones, metadatos, catálogo de objetos y catálogo de representación.

La evolución futura del proyecto contempla, en primer lugar, la armonización con el Mapa Integrado de Centroamérica, acabado en 2012, y la extensión del im-

$2<$ http://geosur.info/arcgis/services/GeoSUR/GeoSUR_MIAN/MapServer/WMSServer?request= GetCapabilities\&service $=$ WMS $>$.

$3<$ http://www.geosur.info/map-viewer/index.html?config=config-rms-es.xml\&lang=es_ES $>$. 
pulso de producción colaborativa de mapas integrados a todo el continente, comenzando con un Mapa Integrado de América del Sur que ya está dando su primeros pasos.

En otro orden de asuntos, se prevé la posible ampliación de recursos disponibles relacionados con el MIAN:

- Un grafo de Hidrografía con topología cadena-nodo, navegable, continuo y por cuencas hidrográficas; un insumo de considerable utilidad del que no hay precedente, por lo que conocemos.

- Un nomenclátor geográfico basado en los objetos considerados en MIAN junto con sus nombres, que permite implementar en la región un servicio de búsqueda por nombre basado en datos oficiales.

- La posibilidad de implementar un servicio de descarga de los datos en un formato abierto y de acuerdo a las licencias y condiciones de cada uno de los Geoinstitutos participantes.

- La implementación de nuevos servicios OGC, como WFS, WCS, WPS, etc., de funcionalidad y posibilidades crecientes.

Las ventajas diferenciales que presenta el MIAN como producto digital de datos geográficos del presente producto provienen en primer lugar de sus fuentes y del carácter de los organismos que lo han producido: son datos geográficos fundamentales y oficiales, fiables, sostenibles y producidos por los Institutos geográficos de la región, que atesoran una larga experiencia y saber hacer en el campo de la producción de cartografía.

En segundo lugar, dado que son fruto de una metodología participativa y de colaboración, están basados en especificaciones logradas mediante el consenso y el libre acuerdo de las partes implicadas, lo que garantiza que el resultado se ha elaborado siguiendo las mejores prácticas en el sector, las recomendaciones más extendidas y los procedimientos más estandarizados y consolidados.

Por otro lado, se ha mantenido como criterio general la conformidad con las normas ISO 19100, las especificaciones OGC y el cumplimiento de las directrices y recomendaciones del IPGH. Adicionalmente se ha aprovechado lo mejor de la experiencia del CNIG en su participación en mapas integrados europeos durante décadas y del USGS en cuanto a la generación de grafos de Hidrología y gestión de imágenes de referencia.

Por último, los Directores y los técnicos de los Institutos geográficos implicados han asumido el compromiso de mantener un periodo de actualización de cinco años, lo que unido al patrocinio del IPGH y CAF, junto a los planes de conexión con el Mapa Integrado de América Central y un futuro Mapa Integrado de América del 
Sur confiere al proyecto las máximas garantías en cuanto a sostenibilidad y mantenimiento de la iniciativa.

De esta iniciativa resultarán beneficiados todos los usuarios de la información geoespacial de la región: tomadores de decisiones, planificadores e investigadores, estudiantes y profesionales. También resultarán beneficiadas otras iniciativas multinacionales, muy en particular la IDE de las Américas y las actividades de UN-GGIM: Américas.

Se trata de una iniciativa que consideramos estratégica, ya que contribuye de manera esencial a la generación y publicación en condiciones abiertas de datos geográficos fundamentales (que en Europa se llaman de referencia) transfronterizos, piedra angular y cimiento fiable de toda la información geográfica que nos rodea, y recurso esencial para afrontar los grandes retos globales del siglo XXI, como el cambio climático y el desarrollo sostenible.

\section{Bibliografía}

Hunt, E. (2012). Informe Técnico del Proyecto IPGH Cart.03.2011, "Integración de datos Mapa Global de América del Sur".

IPGH (2014). Estatuto Orgánico, Reglamentos y Acuerdos 2013-2017, disponible en $<$ https://www.ipgh.org/documentos/ipgh/Estatuto-Organico_2013-2017.pdf $>$.

ISO (2016). Organización Internacional de Normalización ISO/TC211 Programme of work, disponible en $<\mathrm{http}$ ://www.isotc211.org/pow_all.htm>.

Norori, M. (2013). "Proceso participativo de producir un mapa integrado de Centroamérica y sur de México", Revista Cartográfica núm. 89, IPGH.

OGC (2016). Open Geospatial Consortium, "OGC standards and suporting documents", <http://www.opengeospatial.org/standards>.

Proyecto MIAN (2016). “Catálogo de objetos del Mapa Integrado Andino del Norte (MIAN)" (29 de julio de 2016), disponible en <http://www.geosur.info/geosur/ contents/20160729CatalogodeobjetosMIANv2016.pdf $>$.

Proyecto MIAN (2016). "Catálogo de representación del Mapa Integrado Andino del Norte (MIAN)", (29 de julio de 2016), disponible en <http://www. geosur.info/geosur/contents/20160729CatalogodeRepresentacionMIANv2016. pdf $>$.

Proyecto MIAN (2016). "Especificaciones del Mapa Integrado Andino del Norte (MIAN)", (29 de julio de 2016), disponible en <http://www.geosur. info/geosur/contents/20160729EspecificacionesMIANv2016.pdf>.

Rodríguez A.F. (2016). Blog de la IDEE, "Versión beta del MIAN", disponible en $<$ http://blog-idee.blogspot.com.es/2016/07/version-beta-del-mian-disponible.html>. 